\title{
A simple measure to improve the rates of thromboprophylaxis prescription post surgical fixation of neck of femur fractures in a district general hospital
}

\author{
Pranab Sinha, Ali-Asgar Najefi, John Hambidge
}

Queens Hospital, Romford

\begin{abstract}
Neck of femur (NOF) fractures are a major public health concern because of the ageing population and higher incidence of fragility fractures. NOF fractures are associated with high mortality and morbidity rates, and there is a high risk of venous thromboembolism (VTE) after hip fractures (Ref 1). Therefore thromboprophylaxis is vital. Current NICE guidelines advocate $28-35$ days of thromboprophylaxis after NOF fractures (Ref 1,2$)$.
\end{abstract}

It came to our attention that patients post NOF fixation were getting variable prescriptions in regards to their thromboprophylaxis. Therefore a retrospective study on prescription of thromboprophylaxis was conducted from October 2012 to February 2013 within the trauma and orthopaedics department at Queens Hospital, Romford.

Data was collected on all NOF fractures from electronic discharge summaries. Basic descriptive statistics were used to analysis the data. There were 110 cases of NOF fractures during this period. 100 patients were included since two were discounted as they were already on long term anticoagulants and eight patients died in hospital. No thromboprophylaxis was prescribed for 15 patients (15\%). Three patients (3\%) were prescribed less than 28 days (mean 14 days, range (14-14 days)). 69 patients (69\%) received 28 - 35 days of thromprophylaxis, whilst five patients (5\%) received more than 35 days (mean 42 days, range $40-42$ days).

Formal departmental teaching and presentation of the findings was given after the initial study and a small label with the message, 'POST NOF \#: 28-35 days Enoxaparin', was attached to the back of all the junior doctor work phones. After the intervention, data was collected from the period of 7th of January to 7th of February 2013. The second study showed that 50 patients were admitted with NOF fractures in this time period. Four patients died in hospital and three patients were discounted as they were on Warfarin. Two patients were not prescribed thromboprophylaxis (5\%). 34 (79\%) patients received 28 - 35 days, whereas seven patients (16\%) received 42 days of thromboprophylaxis.

The older patients with multiple co-morbidities and reduced mobility are at high risk of developing thromboembolism post NOF fixation. Our initial study identified inadequate prescription of thromboprophylaxis post NOF fractures. After introduction of simple measures such as the reminder label attached to phones, our repeat study found that there was improvement in prescription rates. Our study highlights that simple measures can increase awareness and improve patient safety.

\section{Problem}

Patients post neck of femur fracture (NOF) fixations should get thromboprophylaxis as per NICE guidelines.

During our clinical work, it was observed that prescription rates of thromboprophylaxis varied.

This was obviously concerning as it potentially put our patients at a higher risk of developing venous thromboprophylaxis (VTE). The prescriptions were usually carried out by junior doctors, who may not have been aware of the guidelines and or may have been rotating through the department for a short period of time and hence been unaware of the duration of prescription of thromboprophylaxis in these patients.

\section{Background}

Neck of femur (NOF) fractures are a major public health concern because of the ageing population and higher incidence of fragility fractures. There are 70,000 to 75,000 hip fractures per year with estimated costs of two billion pounds (medical and social) (1). NOF fractures are associated with high mortality and morbidity rates; NICE shows that $10 \%$ of these patients die within one month of their fracture, and $33 \%$ die within 12 months (1). Most of the deaths are related to associated conditions and not the fracture itself indicating a high level of pre-morbid co-morbidities associated with this condition. This is also supported by the American Society of Anesthesiologists (ASA) who reported $70 \%$ of these patients as having a peri-operative anaesthetic severity risk of $3(3)$. More than 25,000 patients die of venous thromboembolism contracted in hospitals each year. This is greater than the combined deaths due to breast cancer, AIDS and road traffic accidents (4).

The introduction of NICE guidelines has eliminated any confusion regarding prophylactic treatment.Complications arising from a 
single VTE can lead to a significant health care burden, costing the health care services more money and time than if they had used thromboprophylaxis in the beginning and complied with guidelines (5). There is also a risk of medico-legal issues and costs if a patient develop VTE and their caregivers have not complied with prophylactic guidelines. As well as providing better patient care, compliance with guidelines also offers some medico-legal protection.

There is a high risk of venous thromboembolism (VTE) after hip fractures (1), and therefore thromboprophylaxis is vital. Current NICE guidelines advocate 28 - 35 days of thromboprophylaxis after NOF fractures (1). Thromboembolism is present in up to $50 \%$ in patient with no thromboprophylaxis (2). We aimed to evaluate whether our department conformed to the NICE guidelines for prescription of thromboprophylaxis in NOF fractures.

\section{Baseline measurement}

A retrospective study on our prescription of thromboprophylaxis was conducted from October 2012 to February 2013 within the trauma and orthopaedics department at Queens Hospital, Romford. An electronic system (ehandover) was used to identify the patients who presented with a NOF fracture. This database captured the demographics, diagnosis, date of admission, operation and discharge, a brief history and their subsequent management. The patients were correlated with the electronic discharge summaries to check whether thromboprophylaxis was correctly prescribed. Basic descriptive statistics were used to analysis the data.

The initial study results showed that there were 110 cases of NOF fractures between 1st of Oct to 31st of Dec 2012. 104 were viable results and further two discounted due to being on long term warfarin. Eight patients died in hospital. There were 35 males and 69 females. No thromboprophylaxis was prescribed for 15 patients. Three patients were prescribed less than 28 days (mean 14 days, range 14 - 14 days). 69 patients received: 28 - 35 days of thromprophylaxis, whilst five patients received more than 35 days (mean 42 days, range $40-42$ days).

\section{Design}

The hypothesis was that the simplest measure which increases awareness will be the most beneficial as it was evident that clinicians can forget guidelines or may not be aware of them.

The best ways to increase awareness was discussed between the authors. Initially formal departmental teaching and presentation of the findings was given after the initial study to raise awareness and to see if our prescription rates improved. The authors anticipated that a 'one off' teaching and presentation of the results may not increase compliance in the prescription rates as clinicians may not be able to readily recall the information presented or may not have been able to attend the presentation meeting. Therefore an informal survey was conducted to test this theory.

\section{Strategy}

It soon became clear through a simple informal survey that though a simple presentation can improve awareness, its role was limited. The survey revealed that junior doctors had a hectic Rota along with the fact that the Rota was dynamic, led to some junior doctors not having been able to attend the presentation and or forgetting the guidelines over time.

Initially, a small poster was displayed in the trauma room to serve as a reminder but the feedback from the junior doctors was that the information was not readily available when they needed to recall the duration of thromboprophylaxis for these patients.

After multiple discussions with senior colleagues and the trauma coordinators (whose role was to assist the trauma team in running a smooth service), a proposal was put by the authors to solve the problem related to recalling the duration of thromboprophylaxis.

The authors suggested devising a small reminder note and affixing that to all the work phones carried by the doctors in the department. The hypothesis being that this will give a quick, accurate reminder which was readily accessible as all junior doctors were assigned their specific work phones.

The department authorised access to all the work phones carried by the medical staff and therefore a small label with the message, 'POST NOF \#: 28-35 days Enoxaparin' was devised and attached to the back of all the junior doctor work phones. After the intervention, data was collected by the same method from the period of in January and February 2013.

\section{Results}

The results from our repeat study showed that during January and February 2013, 50 patients were admitted with NOF fractures. Four patients died in hospital. Three patients were discounted as they were on Warfarin. There were 14 males and 36 females.

Only two patients were not prescribed thromboprophylaxis. 34 patients received: 28 - 35 days of thromprophylaxis. Seven patients received 42 days of thromboprophylaxis.

\section{Lessons and limitations}

Our second study sample size was less compared to the first study sample size. Also the study did not focus on whether there were clinical reasons to deviate from the NICE guidelines for thromboprophylaxis. A control group was not deemed appropriate for the study design.

The department regularly ensures that the label reminder remains. This role is carried out by trauma coordinators.

\section{Conclusion}

An integrated and multifaceted approach to improve prophylaxis rates should be utilised. Simple measures such as asking 


\section{BMJ Quality Improvement Reports}

consultants to remind junior doctors, putting posters up in the trauma doctors office, asking nursing staff to check for a VTE assessment on admission to the ward, and putting reminders on the patient name board, and electronic reminders in hospitals have shown to improve prescription rates of venous thromboprophylaxis for acute orthopaedic admissions (Ref 6). In a systemic review looking at barriers to compliance, Cabana 1999 found that $10 \%$ of physicians were unaware of guidelines (Ref 7). The practice of thromboprophylaxis can be improved by highly influential and respected colleagues (opinion leaders), who can be responsible for disseminating clinical guidelines (Ref 8). A series of trials into the use of risk assessment forms, which were filled in on admission of medical emergencies found that there was a $100 \%$ success rate from completed risk assessment forms to appropriate therapy instituted. This suggests that when used appropriately, risk assessment forms are a highly successful tool in VTE prophylaxis. However, there was a problem with compliance and the forms were not always filled out, leading to inadequate thromboprophylaxis given to the patient (Ref 9).

Our initial study identified inadequate prescription of thromboprophylaxis post NOF fractures. After introduction reminder labels attached to phones, our second study found that there was improvement in prescription rates. Our study highlights that these measures can improve compliance and increase patient safety.

\section{References}

1. NICE guidelines. The management of hip fractures in adults. 2011.

http://www.nice.org.uk/nicemedia/live/13489/54921/54921.p df

2. NICE guidelines. Venous thromboembolism: reducing the risk. 2010

http://www.nice.org.uk/nicemedia/pdf/CG92NICEGuidelineP DF.pdf

3. Sendtner E. Renkawitz T, Kramy P, Wenzl M and Grifka J. Fractured neck of femur - internal fixation vs arthroplasty. Deutches Aerzteblatt International. 2010. 107(23). 401 407

4. House of Commons Health Committee. The Prevention of Venous Thromboembolism in Hospitalised Patients. 2005.

5. Caprini, Joseph A Hyers, Thomas M. Compliance with antithrombotic guidelines. Managed care 2006;15(9):49.

6. Watts $L$ and Grant $D$. Venous thromboembolism (VTE) risk assessment and prophylaxis in acute orthopaedic admissions: improving compliance with national guidelines. BMJ Quallmprov Report. 2013 ;2:2 u202229.w1118

7. Cabana, M D Rand, C S Powe, N R Wu, A W Wilson, M H Abboud, P A Rubin,H R. Why don't physicians follow clinical practice guidelines? A framework for improvement. JAMA 1999;282(15):1458.

8. Kakkar, A K Davidson, B L Haas,S K. Compliance with recommended prophylaxis for venous thromboembolism: improving the use and rate of uptake of clinical practice guidelines. Journal of thrombosis and haemostasis 2004;2(2):221.

9. Sooriakumaran, P Burton, L Choudhary, R Darton, T
Woods, C Lloyd-Mostyn, R H Fernando, D J S Thomson, G A. Are we good at thromboembolic disease prophylaxis - an audit of the use of risk assessment forms in emergency medical admissions. International journal of clinical practice 2005;59(5):605.

\section{Declaration of interests}

Conflicts on interests: None

\section{Acknowledgements}

To our trauma coordinators, Sharon, Joyce and Stuart and our supervising consultant, Mr John Hambidge. 